\title{
PESQUISA DE LISTERIA MONOCYTOGENES EM QUEIJO MUÇARELA FATIADO COMERCIALIZADO EM ESTABELECIMENTOS VAREJISTAS NA CIDADE DE GOIÂNIA, GO
}

Ana Clara Melo Lima, Luiza Chaul Toubas,

Aline Neves Pereira, Geovanna Bárbara Henrique Correia da Silva,

Submetido em: 11/11/2015 leda Maria Sapateiro Torres e Virgínia Farias Alves*

Aceito em: 16/11/2015

Faculdade de Farmácia, Universidade Federal de Goiás

Publicado em: 31/12/2015

*E-mail: valves.ufg@gmail.com

\section{Resumo}

Listeria monocytogenes é o agente causal da listeriose, uma doença de caráter oportunista e grave, que afeta especialmente gestantes, neonatos, idosos e imunodeprimidos. Os alimentos são os principais veículos de transmissão da bactéria, sendo considerados de alto risco especialmente os prontos para consumo, que apresentam longa vida de prateleira sob refrigeração e são consumidos sem aquecimento prévio, como é o caso de diversos derivados lácteos. O objetivo deste trabalho foi verificar a presença de L. monocytogenes em amostras queijo muçarela fatiado, adquiridas no comércio varejista de Goiânia (GO). 34 amostras do produto foram analisadas no Laboratório de Pesquisa em controle de Qualidade de Alimentos e Medicamentos da Faculdade de Farmácia da Universidade Federal de Goiás. As análises foram conduzidas de acordo com o protocolo preconizado pelo Food and Drug Administration. Os resultados foram confirmados com o kit API Listeria. A presença de $L$. monocytogenes foi detectada em quatro $(11,75 \%)$ das 34 amostras de queijo muçarela avaliadas, e a presença de $L$. innocua em uma (2,9\%) das amostras. Apesar de, no Brasil, ainda não ter sido possível estabelecer a relação entre alimentos e doença, os resultados do presente estudo evidenciam que o queijo muçarela fatiado pode atuar como importante veículo para transmissão da bactéria, constituindo, dessa forma, risco para os consumidores.

Palavras-chave: Listeria monocytogenes, alimentos prontos para consumo, laticínios.

\section{Research for listeria monocytogenes in sliced mozzarella cheese sold at retail markets in Goiânia, GO.}

\section{Abstract}

Listeria monocytogenes is the agent of listeriosis, a serious disease with opportunistic character that affects pregnant women, newborns, elderly people and immunocompromised. Foods are the major vehicles to L. monocytogenes transmission, being considered as high risk especially the ones that are ready to eat (RTE), with long shelf life under refrigeration and consumed without heating, as dairy products. The aim of this work was to verify the presence of L. monocytogenes in sliced mozzarella cheese, sold in retail outlets of Goiânia, GO. 34 samples of the product were acquired in supermarkets and bakeries and sent for analysis at the Laboratory for Research on Quality Control of Food and Drugs, Faculty of Pharmacy, Federal University of Goiás. The analyzes were conducted according to the Food and Drug Administration protocol, and the Api Listeria kit was used to confirm the results. The presence of $L$. monocytogenes was verified in four $(11,75 \%)$ from the 34 samples of mozzarella sliced cheese evaluated and $L$. innocua in one (2,9\%). In Brazil, it has not yet been possible to establish the relationship between food and listeriosis. However, the results of this study showed that the sliced mozzarella cheese could act as an important vehicle for transmission of the bacteria, constituting thus risk to consumers.

Key words: Listeria monocytogenes, ready to eat food, Dairy Products. 
Investigación de listeria monocytogenes en queso en lonchas vende en las tiendas minoristas en Goiânia, GO.

\section{Resumen}

Listeria monocytogenes es el agente de la listeriosis, uma enfermedad de naturaleza oportunista y grave que afecta especialmente las mujeres embarazadas, recién nacidos, ancianos e inmunodeprimidos. La comida es el principal vehículo de transmisión de bacterias y se consideran de alto riesgo especialmente listo para el consumo que tienen larga vida útil en condiciones de refrigeración y se comen sin calentamiento, como es el caso de muchos productos lácteos. El objetivo de este estudio fue verificar la presencia de $L$. monocytogenes en muestras de queso mozzarella en rodajas adquiridos en el comercio minorista Goiânia (GO). Se analizaron 34 muestras de productos en el Laboratorio de Investigación para el Control de Calidad de Medicamentos y Alimentos de la Facultad de Farmacia de la Universidad Federal de Goiás. Los análisis se llevaron a cabo de acuerdo con el protocolo recomendado por la Food and Drug Adminstration. Los resultados se confirmaron con el kit API Listeria. Se detectó la presencia de L. monocytogenes en cuatro $(11,75 \%)$ de las 34 muestras queso mozzarella evaluadas y la presencia de $L$. innocua en un (2,9\%) de las muestras. Aunque, en Brasil, aún no ha sido posible establecer la relación entre la alimentación y las enfermedades, los resultados de este estudio muestran que el queso mozzarella en rodajas puede actuar como un importante vehículo para la transmisión de la bacteria y son, por lo tanto, el riesgo para los consumidores.

Palabras clave: Listeria monocytogenes, alimentos listos para el consumo, Productos Lácteos.

\section{INTRODUÇÃO}

As doenças transmitidas por alimentos (DTA), também chamadas de toxi-infecções alimentares, são definidas pela Organização Mundial de Saúde como "doenças de natureza infecciosa ou tóxica causada por, ou através do, consumo de água e alimentos"(1). As DTA representam um grande problema de saúde pública mundial, sendo que, na atualidade, tanto patógenos conhecidos quanto emergentes estão frequentemente envolvidos em surtos de DTA, causando um elevado impacto na saúde pública global. A maioria dos surtos de DTA é resultante do consumo de alimentos contaminados através da manipulação inadequada associada à conservação, transporte, armazenamento ou distribuição em condições impróprias. Assim, micro-organismos patogênicos podem entrar na cadeia alimentar em diferentes etapas do processo. Como são altamente versáteis, podem se adaptar ao ambiente produtivo, conseguindo sobreviver, multiplicar-se e/ou produzir compostos tóxicos.

Dentre as DTA de origem infecciosa, pode-se destacar a listeriose, uma doença de caráter oportunista e grave, associada ao consumo de alimentos contaminados com a bactéria Listeria monocytogenes. Essa doença é responsável por cerca de 2.500 casos de DTA por ano nos Estados Unidos, sendo 500 destes fatais. Embora a morbidade da doença seja baixa (2 a 10 casos por milhão de pessoas), a mortalidade é elevada, pois está associada a uma média de $25 \%$ dos casos de morte por DTA nos EUA, mesmo com tratamento antimicrobiano adequado(2).

Os alimentos são reconhecidos como principal veículo de transmissão de L. monocytogenes para humanos, sendo considerados de alto risco os que oferecem condições para o desenvolvimento da bactéria durante o armazenamento prolongado sob refrigeração e que são prontos para o consumo (APC) sem necessidade de reaquecimento ${ }^{(3,4)}$.

Dentre os APC frequentemente envolvidos em surtos e casos isolados de listeriose, pode-se destacar os produtos lácteos, pois, além de serem ricos em nutrientes, o que favorece o crescimento da bactéria, sua cadeia de produção oferece diversas possibilidades de contaminação(3,5,6). Entretanto, em nosso país, apesar de já haver dados sobre isolamento da bactéria a partir de derivados lácteos, ainda não foi possível estabelecer a relação entre alimentos e doença $(7,8)$. 
No Brasil os queijos de média, alta e muito alta umidade constituem a única categoria de alimentos, com padrão para $L$. monocytogenes. Para esses produtos a legislação estabelece ausência da bactéria em 25 g, em cinco amostras de um mesmo lote ${ }^{(9,10)}$.

O presente estudo teve como objetivo verificar a ocorrência de L. monocytogenes em amostras de queijo muçarela fatiado, adquiridas em estabelecimentos do comércio varejista (padarias e supermercados) localizados em diferentes pontos na cidade de Goiânia-Goiás.

\section{MATERIAIS E MÉTOdOS}

Entre os meses de fevereiro de 2014 e janeiro de 2015 foram adquiridos no comércio local, padarias e supermercados, 34 amostras de queijo fatiado do tipo muçarela. As amostras foram adquiridas no dia do fatiamento e foram transportadas, em suas respectivas embalagens de armazenamento, até o laboratório de Pesquisa em Controle de Qualidade de Alimentos e Medicamentos (LPCQAM), da Faculdade de Farmácia (FF) da Universidade Federal de Goiás (UFG), onde foram imediatamente processadas.

Para a pesquisa L. monocytogenes seguiu-se o protocolo disponibilizado pelo Bacteriological Analytical Manua/(11). Para tanto, a porções de 25 gramas de queijo muçarela fatiado foram adicionados a $225 \mathrm{~mL}$ de caldo de enriquecimento seletivo primário ("Listeria Enrichment Broth" - formulação UVM-1 - Oxoid, Inglaterra) em bolsa plástica estéril (Nasco Whirl-Pak, EUA). A bolsa plástica, contendo caldo UVM-1 com amostra, foi homogeneizada em "Stomacher" (Ethik Technology, Brasil) por dois minutos e, em seguida, incubada à temperatura de $30^{\circ} \mathrm{C}$ por $24 \mathrm{~h}$. Após o período de incubação, transferiu-se $0,1 \mathrm{~mL}$ do caldo UVM1 para um tubo de ensaio contendo $10 \mathrm{~mL}$ de caldo de enriquecimento seletivo secundário - Fraser (Oxoid) - que foi incubado a $35^{\circ} \mathrm{C}$ por 24 - 48h. Dos caldos Fraser que apresentaram coloração enegrecida (presuntivo para Listeria sp.) foi retirada uma alíquota de $0,1 \mathrm{~mL}$ para semeadura em superfície de ágar Oxford e ágar Palcam (Oxoid), que foram incubadas a $35^{\circ} \mathrm{C}$ por 24-48 h. Até cinco colônias características de Listeria sp. observadas nas placas (ágar Oxford - colônias pretas com halo enegrecido; ágar Palcam - colônias esverdeadas com halo enegrecido), foram transferidas com auxílio de alça bacteriológica para placas de ágar Soja Tripticase adicionado de 0,6\% de Extrato de Levedura (TSAYE - Oxoid) e incubadas a $35^{\circ} \mathrm{C}$ por $24 \mathrm{~h}$, para purificação das culturas. Após a purificação das culturas foram realizados testes de catalase e coloração de Gram.

Em seguida, foram realizados testes de motilidade e fermentação de carboidratos. Para a realização destes, uma colônia de cada isolado suspeito para Listeria sp. foi transferida para tubos de ensaio contendo caldo Infusão de Cérebro e Coração (BHI, Oxoid), que foram incubados a $35^{\circ} \mathrm{C}$ por $24 \mathrm{~h}$. Após incubação, 0,1 mL do caldo BHI foram transferidos para tubos de ensaio contendo Purple Broth Base (Difco, Maryland, EUA) adicionado dos seguintes carboidratos na concentração final 0,1\%: dextrose, D-xilose, L-ramnose e D-manitol. Os tubos foram incubados a $35^{\circ} \mathrm{C}$ por até sete dias.

Para o teste de motilidade, a partir do caldo BHI contendo a cultura microbiana, realizou-se a inoculação com o auxílio de uma alça com formato de agulha em meio semi-sólido Motility Test Medium (BBL, EUA), acrescido de 0,3\% de extrato de carne (Oxoid) e incubou-se à temperatura de $25^{\circ} \mathrm{C}$ por até sete dias.

Para a confirmação dos resultados utilizou-se o kit API Listeria (BioMeriéux, França). Uma cepa padrão de L. monocytogenes (ATCC 19117) e uma cepa de L. innocua (ATCC 33090) foram utilizadas como controle para os testes acima descritos.

Os isolados confirmados pelo API Listeria como L. monocytogenes foram congelados a $-20^{\circ} \mathrm{C}$ em caldo $\mathrm{BHI}$ acrescido de 20\% de glicerol (Sigma, Alemanha) para estudos posteriores. 


\section{RESULTADOS E DISCUSSÃO}

Numerosos estudos sobre a ocorrência de L. monocytogenes em diferentes tipos de alimentos ao redor do mundo têm sido relatados. No que concerne aos queijos, a bactéria já foi isolada de muitas variedades em diversos países, inclusive no Brasil, sendo que, surtos de listeriose relacionados a queijos contaminados com a bactéria têm sido relatados desde a década de

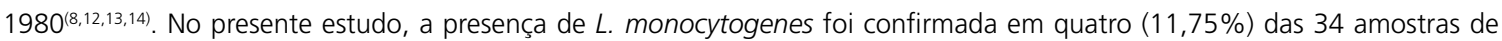
queijo muçarela analisadas e $L$. innocua foi detectada em uma (2,9\%) das amostras avaliadas.

A contaminação por L. monocytogenes nas amostras de queijo muçarela fatiado avaliadas no presente estudo foi mais alta que a encontrada em outros estudos realizados em nosso país, envolvendo não apenas queijos, mas também outros APC, fatiados ou não. Silva et al. (1998) avaliaram a presença de Listeria spp. em queijos tipicamente brasileiros obtidos em mercados varejistas da cidade do Rio de Janeiro e encontraram contaminação por L. monocytogenes em 10,68\% das amostras. Além disso, $12.62 \%$ das amostras estavam contaminadas por L. innocua, $5.83 \%$ por L. grayi e $0.97 \%$ por L. welshimeri(15). Ratti(116), em Ribeirão Preto, SP, analisou 30 amostras de queijo muçarela fatiado, 30 amostras de presunto cozido fatiado e 30 amostras de superfície de equipamento de fatiar. Três $(3,34 \%)$ das 90 amostras avaliadas continham L. monocytogenes e outras três $(3,34 \%)$ continham L. innocua. Dois dos isolados de L. monocytogenes detectados eram provenientes de alimento (presunto) e um do equipamento de fatiar, sendo que o patógeno não foi encontrado em queijo muçarela. Ramos e Costa ${ }^{(17)}$, analisaram 58 amostras de queijo artesanal tipo coalho em Manaus, isolando bactérias do gênero Listeria em apenas duas amostras $(3,4 \%)$, sendo uma da espécie $L$. monocytogenes e uma $L$. innocua. Abrahão et al. ${ }^{(18)}$, analisaram 90 amostras de diferentes tipos de queijo e 60 amostras de sorvete, no estado do Paraná. Nenhuma das amostras de sorvete apresentou contaminação por bactérias do gênero Listeria, enquanto que 12,20\% das amostras de queijo foram positivas para Listeria sp., sendo 6,7\% L. monocytogenes e 5,5\% L. innocua. Martins e Germano(19), em São Paulo, observaram uma maior contaminação por $L$. monocytogenes em amostras de salame $(6,2 \%)$ que em amostras de presunto $(0,8 \%)$. Ainda no âmbito dos APC, Sant'Ana et al. ${ }^{(20)}$, em São Paulo, encontraram contaminação por $L$. monocytogenes em 3,1\% das amostras de verduras e/ou legumes embalados prontos para consumo analisadas.

Os resultados para L. monocytogenes encontrados nesse estudo também foram superiores aos obtidos por diversos pesquisadores que avaliaram queijos e outros APC em diferentes países. Rudolf e Scherer(21) realizaram uma pesquisa com queijos típicos da Europa (red smear cheese) avaliando 329 amostras. Destas, 21 (6,4\%) foram positivas para L. monocytogenes e $39(11,8 \%)$ positivas para outras espécies de Listeria (L. innocua e L. seeligeri). Na Turquia, estudos realizados com queijos tradicionais tipo Herby ${ }^{(22)}$ e tipo Tulum ${ }^{(23)}$ a incidência do patógeno foi de 3,93\% e 4,8\%, respectivamente. Pinto et al. ${ }^{(24)}$, na Itália, detectaram L. monocytogenes em 105 (10\%) de 1045 amostras de APC (produtos de pastelaria, salame, cream cheese, saladas a base de maionese, salmão defumado e queijo muçarela). É importante ressaltar que, das 105 amostras contaminadas por L. monocytogenes, nenhuma era proveniente de queijo muçarela. Um estudo realizado no Reino Unido(25), avaliou 1691 amostras de produtos cárneos fatiados prontos para o consumo e isolou Listeria sp de 45 amostras (2,82\%), sendo que 21 desses isolados $(1,53 \%)$ correspondiam a L. monocytogenes.

Até o momento não foi possível associar o consumo de alimentos contaminados com casos de listeriose no Brasil. Um dos fatores que possivelmente contribui para essa situação é o fato de muitos laboratórios de saúde pública ainda não realizarem rotineiramente a pesquisa desse patógeno em amostras clínicas ou de alimentos, e muitos médicos ainda não estarem alertas para a importância da bactéria ${ }^{(7,26)}$. Além disso, o longo período de incubação da doença dificulta a elucidação de casos de surtos de origem alimentar ${ }^{(7)}$. 
O isolamento de L. monocytogenes a partir de queijo muçarela fatiado, um alimento largamente consumido por brasileiros de todas as classes sociais, é bastante preocupante do ponto de vista de saúde pública, uma vez que os resultados apresentados confirmam que esse alimento pode atuar como importante veículo para transmissão do patógeno.

\section{REFERÊNCIAS}

1. WHO, World Health Organization [cited 2012 June 26]. Food safety and foodborne ilness, 2007. Available from: <http:// www.who.int/mediacentre/factsheets/fs237/en/>

2. Mead PS, Slutsker L, Dietz V, McCaig LF, Bresee JS, Shapiro C, Griffin PM, Tauxe RV. Food-related illness and death in the United States. Emerg Infect Dis 1999; 5(5):607-625.

3. FDA, Food and Drug Administration [cited 2013 May 15]. Quantitative assessment of relative risk to public health from foodborne Listeria monocytogenes among selected categories of ready-to-eat foods. 2003. Available from: <http://www. fda.gov/downloads/food/scienceresearch/researchareas/riskassessmentsafetyassessment/ucm197330.pdf>

4. ILSI Research Foundation, Risk Science Institute. Achieving continuous improvement in reductions in foodborne listeriosis - a risk based approach. J Food Prot 2005; 68(9):1932-94.

5. MacDonald PD, Whitwam RE, Boggs JD, MacCormack JN, Anderson KL, Reardon JW, Saah JR, Graves LM, Hunter SB, Sobel J. Outbreak of listeriosis among Mexican imigrants as result of consumption of illicitly produced mexican-style cheese. Clin Infect Dis 2005; 40:677-82.

6. Gaulin C, Ramsey D, Bekal S. Widespread listeriosis outbreak attributable to pasteurized cheese which led to extensive cross-contamination affecting cheese retailers, Quebec, Canada, 2008. J Food Prot 2012; 75(1):71-8.

7. Brasil. Agência Nacional de Vigilância Sanitária - ANVISA. RDC n 12, de 02 de janeiro de 2001. Aprova regulamento técnico sobre os padrões microbiológicos para alimentos. Brasília (Brasil): 2001.

8. Brasil. Ministério da Agricultura, Pecuária e Abastecimento - MAPA. Instrução Normativa nº 9, de 9 de abril de 2009. Institui os Procedimentos de Controle de Listeria monocytogenes em produtos de origem animal, prontos para o consumo. Brasília (Brasil): 2009.

9. Destro MT. Listeria monocytogenes na cadeia produtiva de alimentos: da produção primária ao consumidor final [thesis]. São Paulo: Faculdade de Ciências Farmacêuticas/USP; 2006.

10. Barancelli GV, Silva-Cruz JV, Porto E, Oliveira CAF. Listeria monocytogenes: ocorrência em produtos lácteos e suas implicações em saúde pública. Arq Instit Biol.2011; 78(1):155-168.

11. BAM - Bacteriological Analytical Manual. [cited 2014 May 18]. Detection and Enumeration of Listeria monocytogenes in Foods. April, 2011. Available from: <http://www.fda.gov/food/foodscienceresearch/laboratorymethods/ucm071400.htm>

12. Pintado CMBS, Oliveira A, Pampulha ME, Ferreira MASS. Prevalence and characterization of Listeria monocytogenes isolated from soft cheese. Food Microbiol 2005; 22(1):79-85.

13. Hof H. History and epidemiology of listeriosis. FEMS Immunol Med Microbiol 2003; 35(3):199-202. 
14. Lianou A, Sofos JN. A review of the incidence and transmission of Listeria monocytogenes in ready-to-eat products in retais and food service environments. J Food Protect 2007;70(9):2172-2198.

15. Da Silva MCD, HOFER E; TIBANA A. Incidence of Listeria monocytogenes in cheese produced in Rio de Janeiro, Brazil. J Food Protect 1998; 3:354-356.

16. Ratti RP. Listeria monocytogenes em alimentos fatiados e equipamentos: ocorrência, formação de biofilme e controle [dissertation]. Ribeirão Preto: Faculdade de Ciências Farmacêuticas/USP; 2006.

17. Ramos SNM, Costa CA. Ocorrência de Listeria monocytogenes em queijo artesanal tipo coalho comercializado na cidade de Manaus-AM, Brasil. Acta Amazon 2003; 33(4):613-618.

18. Abrahão WM, Abrahão PRS, Monteiro CLB, Pontarolo R. Occurrence of Listeria monocytogenes in cheese and ice cream produced in the State of Paraná, Brazil. Braz J Pharmaceut Sci. 2008; 44(2):125-129.

19. Martins EA. Listeria monocytogenes em produtos fatiados do tipo ready-to-eat, presunto cozido e salame, comercializados no município de São Paulo: Ocorrência, quantificação e sorotipagem [thesis]. São Paulo: Faculdade de Saúde Pública/USP; 2010.

20. Sant'ana AS, Igarashi MC, Landgraf M, Destro MT, Franco BDGM. Prevalence, populations and pheno- and genotypic characteristics of Listeria monocytogenes isolated from ready-to-eat vegetables marketed in São Paulo, Brazil. Int J Food Microbiol 2012; 155: 1-9.

21. Rudolf M, Scherer S. High incidence of Listeria monocytogenes in European red smear cheese. Int J Food Microbiol 2001; 63(11):91-98.

22. Sagun E, Sancak YC, Isleyici Ö, Ekici K. The presence and prevalence of Listeria species in milk and Herby cheese in and around Van. Turk J Vet Anim Sci 2001; 25:15-19.

23. Colak H, Hampikyan H, Bingol EB, Ulusoy B. Prevalence of L. monocytogenes and Salmonella spp. in Tulum cheese. Food Control 2007; 18(5):576-579.

24. Di Pinto A, Novello L, Montemurro F, Bonerba E, Tantillo G. Occurrence of Listeria monocytogenes in ready-to-eat foods from supermarkets in Southern Italy. New Microbiol 2010; 33(2):249-252.

25. FSA, Food Standards Agency [cited 2014 June 27]. A microbiological survey of retail ready-to-eat cooked sliced meats and pâtés with particular reference to the presence of Listeria monocytogenes. 2011. Available from: <http://multimedia.food. gov.uk/multimedia/pdfs/fsis0111.pdf>

26. Hofer $E$, Reis CMF, Hofer CB. Sorovares de Listeria monocytogenes e espécies relacionadas, isoladas de material clínico humano. Rev Soc Bras Med Trop 2006; 39(1):32-37.

Agência de Fomento: Fundação de Amparo à Pesquisa do Estado de Goiás (FAPEG). 